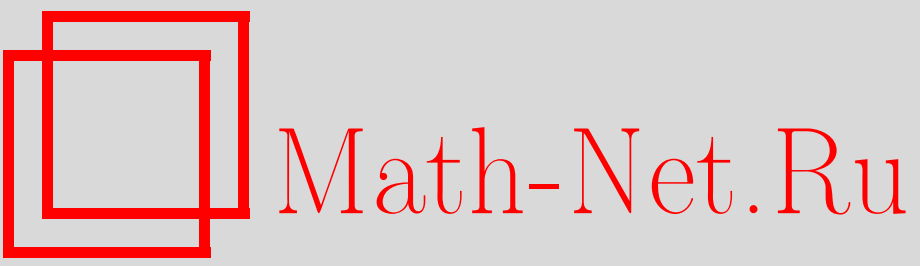

Е. К. Лейнартас, М. Пассаре, А. К. Цих, Асимптотика многомерных разностных уравнений, УМН, 2005, том 60, выпуск 5, 171-172

DOI: https://doi.org/10.4213/rm1649

Использование Общероссийского математического портала Math-Net.Ru подразумевает, что вы прочитали и согласны с пользовательским соглашением

http://www . mathnet.ru/rus/agreement

Параметры загрузки:

IP: 54.237 .206 .68

26 апреля 2023 г., 16:45:34 


\title{
АСИМПТОТИКА МНОГОМЕРНЫХ РАЗНОСТНЫХ УРАВНЕНИЙ
}

\author{
Е. К. ЛЕЙНАРтАС, М. ПАССАРЕ, А. К. ЦИХ
}

Пусть $f(x)$ - комплекснозначная функция дискретного аргумента $x \in \mathbb{Z}^{n}$. На векторном пространстве таких функций рассмотрим $n$ операторов сдвига $\delta_{j} f(x)=f\left(x+e_{j}\right), j=1, \ldots, n$ $\left(e_{j}\right.$ - базисные орты). С помощью набора $\delta=\left(\delta_{1}, \ldots, \delta_{n}\right)$ поставим в соответствие каждому полиномиальному символу $P(x, z)=\sum_{\alpha} a_{\alpha}(x) z^{\alpha}$ с переменными коэффициентами скалярньй разностный оператор $P(x, \delta) f(x)=\sum_{\alpha} a_{\alpha}(x) f(x+\alpha)$. В работе изучено асимптотическое поведение при $x \rightarrow \infty$ решений систем разностных уравнений

$$
P_{1}(x, \delta) f(x)=\cdots=P_{n}(x, \delta) f(x)=0,
$$

а также скалярных разностных уравнений с постоянными коэффициентами

$$
P(\delta) f(x)=0 .
$$

Одномерный случай $(n=1)$ был изучен еще в фундаментальной работе Пуанкаре [1]. В частности, было доказано, что если старший коэффициент $a_{k}(x)$ разностного уравнения порядка $k$ нормирован условием $a_{k}(x) \equiv 1$, а все остальные коэффициенты имеют конечные пределы при $x \rightarrow \infty$, причем все характеристические корни $\lambda_{1}, \ldots, \lambda_{k}$ предельного уравнения различны по модулю, то для любого ненулевого решения $f(x)$ уравнения предел $\lim _{x \rightarrow \infty} f(x+1) / f(x)$ существует и равен одному из характеристических корней $\lambda_{j}$.

Для формулировки первого многомерного обобщения упомянутого резултата Пуанкаре отметим, что возможность нормирования старшего коэффициента уравнения в многомерном случае соответствует условию: проекция $\pi:\left\{(x, z) ; P_{1}(x, z)=\cdots=P_{n}(x, z)=0\right\} \rightarrow \mathbb{C}_{x}^{n}$ должна быть собственным отображением.

С функцией $f$ ассоциируем вектор $\left(f\left(x+e_{1}\right) / f(x), \ldots, f\left(x+e_{n}\right) / f(x)\right)$, который назовем вектором Горна (в честь автора, применившего его в определении общего гипергеометрического ряда).

ТЕОРема 1. Предположим, что проекция $\pi$ собственная и что для данного направления $q \in \mathbb{Z}^{n} \backslash\{0\}$ каждый из коэффичиентов системь (1) стремится $к$ конечному пределу при $x \rightarrow \infty$ вдоль любой последовательности вида $\{a+q l ; l \in \mathbb{N}\}$, где $a \in \mathbb{Z}^{n}$ фиксированные векторы. Если для корней $\lambda_{(1)}, \ldots, \lambda_{(k)}$ предельной характеристической системь, соответствующей (1), мономь $\lambda_{(1)}^{q}, \ldots, \lambda_{(k)}^{q}$ различны по абсолютной величине, то для любого ненулевого решения $f(x)$ системы (1) предел вектора Горна

$$
\left.\lim _{l \rightarrow \infty}\left(f\left(x+e_{1}\right) / f(x), \ldots, f\left(x+e_{n}\right) / f(x)\right)\right|_{x=q l}
$$

существует и равен одному из характеристических корней $\lambda_{(p)}$ предельной системь.

Доказательство основано на обобщенной теореме Вейерштрасса о делении на идеал в кольце полиномов и на обобщении определителя Вандермонда для корней $\lambda_{(1)}, \ldots, \lambda_{(k)}$.

Применительно к уравнению (2) использется понятие амебы (см. [2], [3]) характеристического многочлена $P(z)$, т.е. образ множества нулей $V=\{P(z)=0\}$ при логарифмическом проектировании $\log :\left(z_{1}, \ldots, z_{n}\right) \mapsto\left(\log \left|z_{1}\right|, \ldots, \log \left|z_{n}\right|\right)$. Будем обозначать амебы через $\mathscr{A}_{V}$. Дополнения $\mathbb{R}^{n} \backslash \mathscr{A}_{V}$ состоят из конечного числа связных компонент $\{E\}$, каждая из которых открыта и выпукла [3]. Далее, число компонент $\mathbb{R}^{n} \backslash \mathscr{A}_{V}$ не больше числа целых точек многогранника Ньютона $N_{P}[2]$.

Все три автора были поддержаны шведскими фондами им. Густавсона и Королевской Академии наук. Первый автор также был поддержан грантом Президента РФ НШ-1212.2003.1, а третий автор - грантом РФФИ 02-01-00167. 
Нас интересуют экстремальные амебы, для которых в дополнении $\mathbb{R}^{n} \backslash \mathscr{A}_{V}$ имеется максимальное число $\#\left\{\mathbb{Z}^{n} \cap N_{P}\right\}$ связных компонент. В одномерной ситуации этот случай соответствует тому, что все корни имеют попарно различные модули.

Класс допустим ых решений уравнения (2), которые мы будем рассматривать, дается интегралами

$$
f(x)=\int_{\gamma} z^{x} \omega(z), \quad x \in \mathbb{Z}^{n},
$$

где $\omega \in \Omega^{n-1}(V)$ - голоморффные $(n-1)$-формы на $V$, а $\gamma$ - $(n-1)$-циклы на $V$. Другие решения, как правило, ведут себя хаотически и не поддаются асимптотическому контролю.

Теорема 2. Предположим, что амеба $\mathscr{A}_{V}$ характеристического множества уравнения (2) имеет гладкую границу и что $V \cap \log ^{-1}(x)$ состоит из одной точки для каждого $x \in \partial \mathscr{A}_{V}$. Тогда для любого ненулевого допустимого решения (3) и для почти любого направления вектора $q \in \mathbb{Z} P_{n-1}$ по крайней мере один из пределов

$$
\left.\lim _{k \rightarrow \pm \infty}\left(f\left(x+e_{1}\right) / f(x), \ldots, f\left(x+e_{n}\right) / f(x)\right)\right|_{x=k q}
$$

существует и равен точке $z \in V$.

Следующее утверждение показывает, что класс двумерных характеристических множеств, удовлетворяющих условию теоремы, составляют кривые Гарнака [4].

Tеорема 3. Пусть $n=2$. Предположим, что амеба $\mathscr{A}_{V}$ әкстремальная и что граница д $\mathscr{A}_{V}$ гладкая. Тогда $V \cap \log ^{-1}(x)$ coстоит из одной точки для каждого $x \in \partial \mathscr{A}_{V}$. В частности, вещественные алгебраические кривые, у которых амебы әкстремальные и с гладкими границами, являются гладкими гарнаковскими кривыми.

При доказательстве теоремы 2 используется специальная функция Морса на $V$, равная $\mid z^{q} \|_{V}$. Для любого направления $q$ ее критические точки совпадают с прообразом логарифмического отображения Гаусса [5]. Затем показывается, что для почти всех $q$ функция $\mid z^{q} \|_{\gamma}$ принимает минимальное значение в единственной точке цикла $\gamma$. Поскольку эта функция служит фазой осциллирующего интеграла (3), дело сводится к методу перевала. В доказательстве теоремы 3 применяется известная теорема Пика о связи числа целых точек в многоуголнике и его площади, а также свойства логарифмического отображения Гаусса.

\section{СПИСОК ЛИТЕРАТУРЫ}

[1] H. Poincaré // Amer. J. Math. 1885. V. 7. P. 203-258. [2] M. Forsberg, M. Passare, A. Tsikh // Adv. Math. 2000. V. 151. № 1. P. 45-70. [3] I. M. Gelfand, M. M. Kapranov, A. V. Zelevinsky. Discriminants, Resultants and Multidimensional Determinants. Boston: Birkhäuser, 1994. [4] G. Mikhalkin, H. Rullgård // Internat. Math. Res. Notices. 2001. № 9. P. 441-451. [5] M. Passare, A. Tsikh // Contemp. Math. 2005. V. 377. P. 275-288.

Красноярский государственный университет; Matematiska Institutionen, Stockholms Universitet; Красноярский государственный университет

E-mail: lein@mail.ru; passare@math.su.se; tsikh@lan.krasu.ru
Представлено В.М. Бухштабером Принято редколлегией 22.07.2005 